\title{
Respiratory muscle strength and endurance in individuals with tetraplegia
}

\author{
MTE Hopman ${ }^{1}$, LHV van der Woude $^{2}$, AJ Dallmeijer ${ }^{2}$, G Snoek ${ }^{3}$ and HTM Folgering ${ }^{4}$ \\ ${ }^{1}$ University of Nijmegen, ${ }^{2}$ Free University of Amsterdam, ${ }^{3}$ St Maartenskliniek, Rehabilitation Center Nijmegen, \\ ${ }^{4}$ Medical Pulmonary Center Nijmegen, The Netherlands
}

\begin{abstract}
The purpose of this study was to assess the strength and endurance capacity of the respiratory muscles in individuals with tetraplegia and to compare these properties to those in able-bodied subjects. In addition, the relationship between respiratory muscle properties and respiratory function, ie, ventilation and gas exchange, was examined. Fifteen individuals with tetraplegia (TP) and 15 able-bodied controls (AB) participated in this study. Respiratory muscle strength was assessed by measuring static inspiratory (Pi-max) and expiratory (Pe-max) mouth pressure, whereas inspiratory endurance capacity (Pendu) was determined using an incremental ventilatory threshold loading test. Results were significantly lower in TP compared to AB: Pi-max (5.9 vs $8.4 \mathrm{kPa})$, Pe-max $(5.6$ vs $12.3 \mathrm{kPa})$, Pendu (2.7 vs $6.9 \mathrm{kPa})$, vital capacity $(3.1$ vs $5.5 \mathrm{l})$ and rest oxygen uptake $(0.21$ vs $0.29 \mathrm{ml} / \mathrm{min})$ and significant correlations were found between the respiratory muscle properties and the respiratory function. Of note is the fact that the ratio Pendu/Pi-max was significantly lower in TP $(0.49)$ compared to $\mathrm{AB}$ (0.82). This implies that the capacity to generate pressure during the endurance test was not fully used in TP, probably due to an early onset of muscle fatigue and an altered force-length relationship of the diaphragm muscle. Results of this study demonstrate a limited ability in individuals with tetraplegia to ventilate.
\end{abstract}

Keywords: respiratory muscle fatigue; respiratory muscle weakness; static mouth pressure; incremental threshold loading; spinal cord injury; tetraplegia

\section{Introduction}

A severe spinal-cord injury (SCI) results in loss of motor innervation of the muscles below the lesion and concomitant muscle atrophy and weakness. Until now most of the research has been focused on establishing strength and fatigue of arm and leg muscles and on optimizing rest-function for ambulation. $5,9,12,13$

Individuals with tetraplegia (TP) experience varying degrees of paralysis of respiratory muscles and for these individuals there is a need to improve respiratory muscle function for optimal ventilation and gas exchange, necessary for daily life and sport activities. In addition, a disfunction of respiratory muscles, leading to a disability to cough up phlegm, increases the risks for pulmonary diseases such as pneumonia. ${ }^{4}$

However, before training the muscles to optimize respiratory rest-function in these individuals, it is important to assess respiratory muscle strength (weakness) and endurance (fatigue) in order to develop an efficacious training program and to be able to evaluate the effect of the training properly. From research related to pulmonary rehabilitation it is known that inspiratory and expiratory muscle strength can be measured accurately using static maximal

Correspondence: MTE Hopman, M.D., Ph.D. inspiratory and expiratory mouth pressure tests, respectively. ${ }^{11,14,15,18}$ The ventilatory incremental threshold loading test has shown to be suitable to measure endurance capacity of the inspiratory muscles. ${ }^{7,17}$ These methods, however, have never been applied to qualify or quantify respiratory muscle function in individuals with tetraplegia.

The purpose of this study, therefore, was to assess respiratory muscle strength (weakness) and endurance capacity (fatigue) in individuals with tetraplegia in comparison to able-bodied subjects using well-established respiratory muscle tests. In addition, the relationship between respiratory muscle properties and respiratory function (ie ventilation and gas exchange at rest and during exercise) in individuals with tetraplegia was examined.

\section{Methods}

\section{Subjects}

Fifteen male individuals with tetraplegia (TP) and 15 male able-bodied controls (AB), matched for age, body mass and physical activity-level, participated in this study. The lesion levels in TP varied between $\mathrm{C} 4$ and $\mathrm{C} 7$ and the lesions existed from 1 to 11 years. Three 
individuals had an incomplete (one at $\mathrm{C} 4$ and two at C5; Frankel score D [2] and C) and 12 had a complete lesion (one at $\mathrm{C} 4$, seven at $\mathrm{C} 5$, one at $\mathrm{C} 6$ and seven at C5). The working muscles varied accordingly to the lesion level, from the diaphragm muscle only (C4) to the scaleus, pectoralis, latissimus dorsi and serratus anterior muscles (C5-C8). None of the subjects was wearing an abdominal binder during the tests. Eight individuals in TP were untrained (less than $1 \mathrm{~h}$ of sport activities per week), whereas the other seven were trained ( 2 to $5 \mathrm{~h}$ of sport activities per week) for more than 2 years. AB were low to moderately trained (1 to $7 \mathrm{~h}$ of sport activities per week).

The medical status of TP and AB was assessed by interview. None of the subjects had any cardiovascular or pulmonary disease or used medications likely to affect the results of the study. The study was approved by the Faculty Ethical Committe, and all subjects gave their written informed consent.

\section{Protocol}

$\mathrm{AB}$ and TP visited the laboratory one time to perform the maximal mouth pressure tests and the incremental threshold test. On the same occasion slow inspiratory vital capacity (iVC) and oxygen uptake at rest $\left(\mathrm{VO}_{2}\right.$ rest) were measured. TP visited the laboratory a second time to perform a maximal arm-cranking exercise test in order to assess maximal oxygen uptake $\left(\mathrm{VO}_{2} \mathrm{max}\right)$ and minute ventilation (VEmax). Temperature in the testing-room was kept between 20 and $21^{\circ} \mathrm{C}$ with a relative humidity of 38 to $43 \%$. All subjects refrained from nicotine, caffeine and alcohol at least $2 \mathrm{~h}$ prior to the tests.

\section{Material and measurements}

The static maximal mouth pressure test

To measure static maximal inspiratory (Pi-max) and expiratory (Pe-max) mouth pressure a plastic flanged mouthpiece was connected to a closed, rigid, plastic tube with a small leak (ID $1.8 \mathrm{~mm}$; length $40 \mathrm{~mm}$ ), the latter to prevent buccal muscles from producing significant pressures and closing of the glottis. ${ }^{15,18}$ The pressure inside the tube was measured with a pressure transducer (Statham USA Gould P23ID) connected to a pressure monitor (Statham USA Gould SP1405) and results were recorded on a chart-recorder (Kipp \& Zonen BD41, Delft, The Netherlands). The pressure system was calibrated before each experiment. The subjects were in a sitting position, wearing a noseclip and breathing through the mouthpiece which was fixed to a standard. Pi-max was measured after maximal expiration at residual volume (RV), whereas Pe-max was measured after maximal inspiration at total lung capacity (TLC). After a short familiarization each subject repeated the manoeuvre at least three times until two almost identical readings were obtained (within 5\% difference). All subjects were verbally encouraged by the examiner and received visual feedback from a chart-recorder. Maximum pressures had to be maintained for at least $1 \mathrm{~s}$ (plateau) to qualify the attempt as being valid. The highest plateaupressure value was used for analysis as being the maximal mouth pressure and, thus, representing maximal respiratory muscle strength. ${ }^{2,14,22}$

One minute incremental threshold loading test

Following a $20 \mathrm{~min}$ recovery-break, the endurance capacity or fatiguability of the inspiratory muscles was assessed using the $1 \mathrm{~min}$ incremental threshold loading test. ${ }^{17} \mathrm{~A}$ modified breathing valve was used, in which weights could be added to the inspiratory valve to change the threshold pressure without disrupting the system or the breathing pattern. The pressure needed to lift the inspiratory valve was measured with a pressure transducer (Statham USA Gould P23ID), connected to a pressure monitor (Statham USA Gould SP1405) and results were recorded on a chart-recorder (Kipp \& Zonen BD41, Delft, The Netherlands).

All subjects were in a sitting position and started to breathe with no load. Every minute weights were added, varying between 10 and $50 \mathrm{~g}$ depending on the achieved Pi-max in the former test. The test used a continuously increasing protocol and was terminated when the subject was not able to lift the inspiratory valve any more. The pressure achieved with the heaviest load (tolerated for $1 \mathrm{~min}$ ) was defined as the Endurance Pressure (Pendu). Pendu was expressed as a percentage of Pi-max [(Pendu/Pi-max $)^{*} 100 \%$, the so called Relative Pendu (RPendu).

\section{Resting metabolic values}

Resting metabolic values were measured for $5 \mathrm{~min}$ after a $15 \mathrm{~min}$ period of absolute rest. $\mathrm{VO}_{2}$ rest and minute ventilation at rest (VErest) were taken as the mean of the last minute. Slow inspiratory Vital Capacity (iVC) was measured three times with a respiratory gas meter (Respiration Gasmeter, Max Planck Institute for Work Physiology, Dortmund, Germany). The average of the three measurements was taken as iVC.

\section{Maximal exercise test}

For TP, $\mathrm{VO}_{2} \max$ and VEmax were assessed during a maximal arm-cranking test using a continuously increasing protocol ( 2 to 5 Watt per min). Exercise was performed on an electrically braked arm-crank ergometer (modified cycle ergometer, Lode, Groningen, The Netherlands) and the hands were fixed to the handles using specially designed mitts. The test was terminated when the cycle frequency fell below $60 \mathrm{rpm}$. The subjects were verbally encouraged during the whole test. $\mathrm{VO}_{2} \max$ and VEmax were calculated as the mean of the last minute.

Ventilation and gas exchange values were measured 
using an automatic gasanalyzer (Oxycon IV, Mijnhardt, Bunnik, The Netherlands), which was calibrated before each test with known gas mixtures.

\section{Statistical analysis}

A Student's $t$ test was applied to assess differences in physical and respiratory characteristics between TP and AB. Differences in respiratory characteristics within TP related to completeness of the lesion or training status were assessed using a Wilcoxon non-parametric rank test. Statistical significance was set at $P<0.05$.

The Spearman correlation coefficient was used to determine correlations between respiratory muscle strength and endurance with $\mathrm{iVC}, \mathrm{VO}_{2}$ rest, $\mathrm{VO}_{2} \max$ and VEmax. To protect against a type I error, a $P<0.01$ was considered being statistically significant.

\section{Results}

Physical characteristics were not significantly different between TP and AB (Table 1). Pe-max, Pi-max, Pendu, iVC and $\mathrm{VO}_{2}$ rest were significantly lower in TP compared to $\mathrm{AB}$ (Figures 1 and 2). RPendu was significantly lower in TP $(49 \%)$ compared to AB $(82 \%)$ (Figure 1).

No significant differences in respiratory muscle characteristics were found between complete and incomplete TP nor between trained and untrained TP. However, a trend can be noticed between a higher training status and a better respiratory muscle function (Figure 3).

Significant correlations $(P<0.001)$ were found in TP between respiratory muscle properties and respiratory function: between Pe-max and iVC, Pe-max and Pi$\max , \mathrm{VO}_{2}$ rest and $\mathrm{iVC}, \mathrm{VO}_{2} \max$ and $\mathrm{iVC}$ and between $\mathrm{VO}_{2} \mathrm{max}$ and Pe-max.

\section{Discussion}

Although it is well known that respiratory muscle function is of life-importance as well as important for daily-life in humans, only a limited number of studies have been directed towards assessment and improvement of respiratory muscle function in individuals with spinal cord injury. In this study an attempt is made to assess respiratory muscle function in an adequate and

Table 1 Physical characteristics of individuals with tetraplegia (TP) and able-bodied controls (AB)

\begin{tabular}{lcc}
\hline & $\begin{array}{c}T P \\
(\mathrm{n}=15)\end{array}$ & $\begin{array}{c}A B \\
(\mathrm{n}=15)\end{array}$ \\
\hline Age (y) & $28.9 \pm 8.8$ & $25.2 \pm 3.1$ \\
Body mass (kg) & $74.6 \pm 13.9$ & $76.5 \pm 7.7$ \\
Height (cm) & $184.4 \pm 8.9$ & $182.6 \pm 6.2$ \\
Smokers (n) & 3 & 5 \\
\hline
\end{tabular}

$n=$ the number of subjects. Values are mean \pm standard deviation (SD)

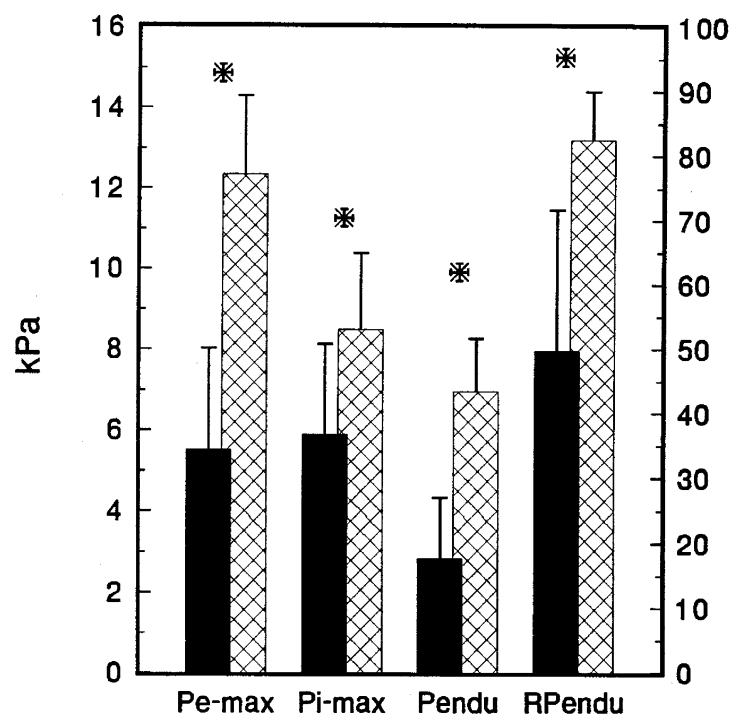

๖

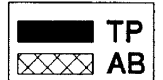

Figure 1 Pe-max, Pi-max and Pendu in $\mathrm{kPa}$ and RPendu in $\%$ for individuals with tetraplegia (TP) and able-bodied controls $(\mathrm{AB})$. Pe-max $=$ maximal static expiratory mouth pressure, $\mathrm{Pi}-\max =$ maximal static inspiratory mouth pressure, Pendu $=$ pressure at the end of the endurance test for inspiratory muscles, RPendu=pressure at the end of the endurance test expressed as a percentage of the maximal inspiratory pressure. Values are mean \pm SD. $*=$ statistically significant, $P<0.05$

reliable way using well-established methods from pulmonary function assessment in individuals with tetraplegia in comparison to able-bodied controls.

The values of strength and endurance capacity of the respiratory muscles in $\mathrm{AB}$ found in this study are in agreement with values reported in previous studies, ${ }^{16,21,22}$ however, considerably lower in comparison to some other studies. ${ }^{1,20}$ The latter disparity can probably be explained by differences in methods used in these studies: (i) a difference in air leak may lead to a difference in generated pressure, ${ }^{18}$ (ii) the type of mouthpiece will influence the generated pressure, ie, a tube shaped mouthpiece generates a higher pressure than a flanged mouthpiece ${ }^{15}$ and (iii) the way the mouthpiece is presented to the subject may also influence the generated pressure, ie, a mouthpiece held by the subject, instead of one fixated in a standard like in the present study, appeared to generate higher pressures. ${ }^{15}$

Since, in the present study strength and endurance capacity of respiratory muscles in $\mathrm{TP}$ and $\mathrm{AB}$ were measured by the same method, a comparison between the two groups will not be influenced by the above mentioned factors. 


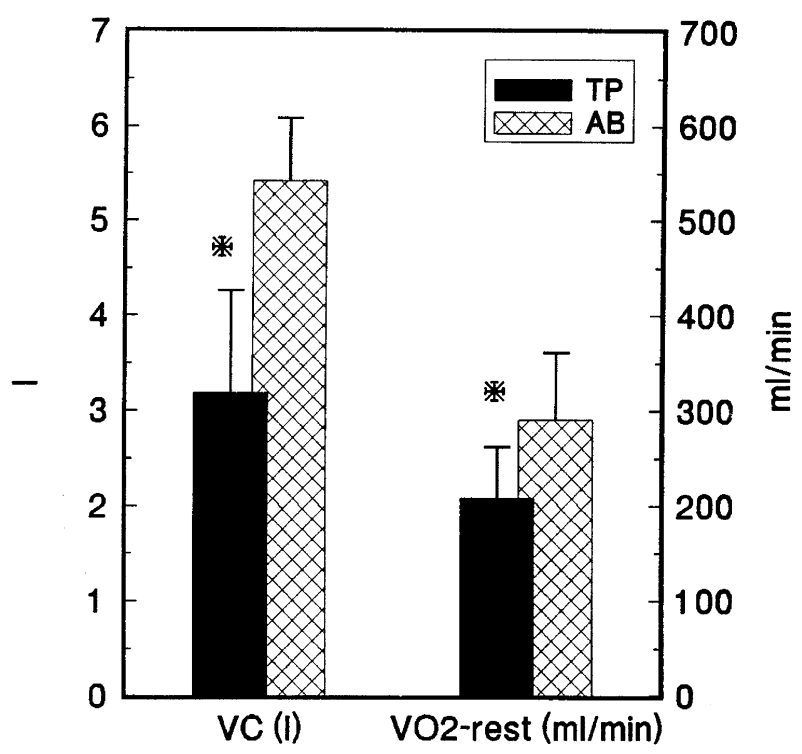

Figure 2 Vital Capacity (VC) in 1 and oxygen uptake $\left(\mathrm{VO}_{2}\right)$ in $\mathrm{ml} / \mathrm{min}$ for individuals with tetraplegia (TP) and ablebodied controls $(\mathrm{AB})$ at rest. All values are mean $\pm \mathrm{SD}$. $*=$ statistically significant, $P<0.05$
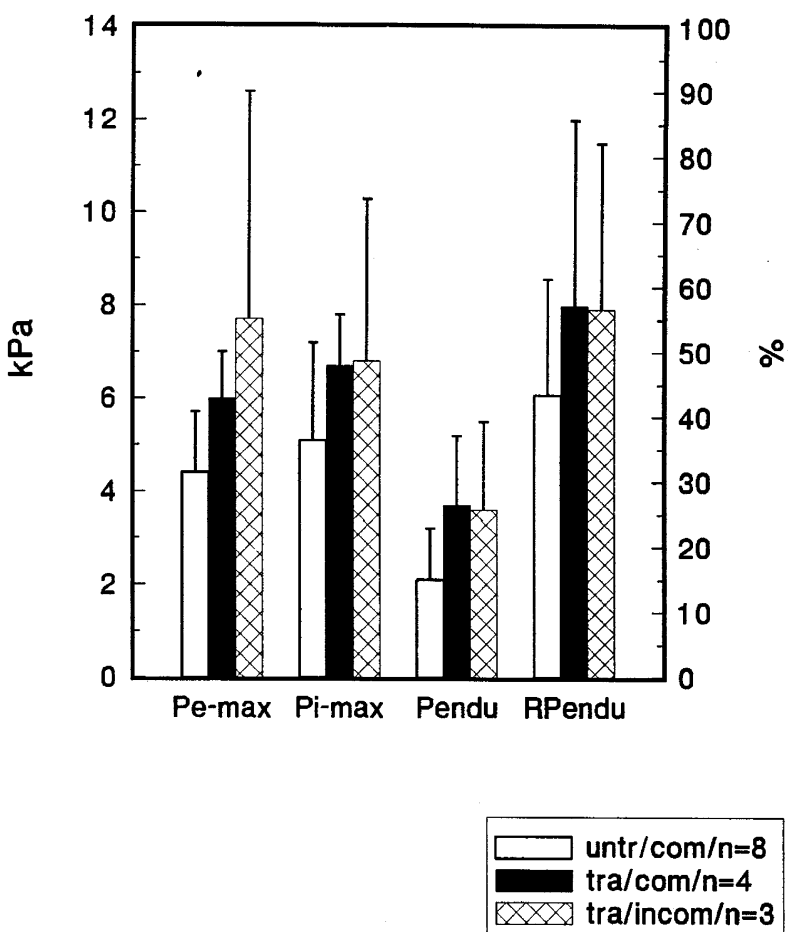

Figure 3 Maximal static expiratory mouth pressure (Pe-max in $\mathrm{kPa}$ ), maximal static inspiratory mouth pressure (Pi-max in $\mathrm{kPa}$ ), pressure at the end of the endurance test for inspiratory muscles (Pendu in $\mathrm{kPa}$ ) and Relative endurance capacity (RPendu in \%) for individuals with tetraplegia divided into groups based on completeness of the lesion and training status (untr/com $=$ untrained status and complete lesion, tra/ com $=$ trained status and complete lesion, tra/incom $=$ trained status and incomplete lesion)
Static mouth pressure test: Pi-max and Pe-max

The lower static mouth pressure in TP compared to $\mathrm{AB}$ is likely to result from the denervation of the respiratory muscles in $\mathrm{TP}$, who have only a small muscle mass available for ventilation and to generate pressure.

\section{One-minute ventilatory threshold loading test; Pendu and} RPendu

The lower Pendu in TP suggests an early onset of muscle fatigue in these individuals compared to AB. As a result of respiratory muscle paralyses and atrophy in TP, compensatory activity of the innervated and working muscles will lead to early onset of fatigue. Of note, however, is that even RPendu is markedly lower in TP compared to AB. This implies that the capacity to generate pressure during the endurance test is not fully used in TP, likely as a result of a very early onset of muscle fatigue due to the limited capacity of strength and endurance of the working muscles based on a relatively sedentary life style. ${ }^{10}$ In addition, the possibility of an altered force-length relationship of the diaphragm muscle in TP may account partly for a lower endurance capacity of the inspiratory muscles in these individuals. Finally, the low endurance capacity however, results indicate that training should be focused on improvement of endurance capacity of the inspiratory muscles in TP and it can be hypothesized that this may improve respiratory function in TP.

Inspiratory vital capacity and $\mathrm{VO}_{2}$ rest

The differences found in iVC between TP and AB can be explained in two ways: first, irreversible atelectasis and changes in elastic properties of the lungs may have occurred in TP leading to higher levels of RV. Secondly, the limited respiratory-muscles function, such as paralysis of the abdominal and intercostal muscles and the severe decrease in endurance of the innervated respiratory musculature, will contribute to a higher RV and, consequently, a lower iVC. ${ }^{8}$ The changes in elastic property of the lung tissue will influence the Pe-max, which may explain the correlation between VC and Pe-max. The lower $\mathrm{VO}_{2}$ rest in TP may be due to a pronounced muscle atrophy of the legs, trunk and partly of the arms caused by the motoric denervation based on the spinal cord injury, which means a loss of considerable mass of oxygen consuming tissue in TP.

\section{Completeness of the lesion and training status}

The fact that neither training status nor completeness of the lesion had any significant influence on the respiratory muscle characteristics, may partly be explained by the rather small and varying number of subjects in each group. However, a trend can be noticed in training status and generated static mouth pressure (Figure 3). Gross et $a l^{10}$ trained the 
diaphragm in TP and found improvements which would be compatable with this trend. Additional research is needed to elucidate this issue and to examine the benefits of respiratory muscle training for daily life and the medical status in TP.

\section{Correlations}

The correlations found in this study between the static mouth pressure and other respiratory characteristics are most likely the result of the involvement of the same muscles in these measurements. The degree of paralysis of the respiratory muscles will influence Pemax, Pi-max and $\mathrm{VC}$ as has been mentioned and outlined before. In addition, the available active muscle mass will affect $\mathrm{VO}_{2}$ rest and $\mathrm{VO}_{2}$ max.

Future studies should be directed towards the trainability of respiratory muscles in TP, the benefits for daily life activities, exercise performance and the effect on the prevalence of lung diseases. In particular the expiratory muscle properties and function has to elucidated further because these muscles are severely affected and they play a pivotal role in the positioning of the diaphragm.

\section{Conclusion}

Respiratory properties such as Pi-max, Pe-max, Pendu, $\mathrm{RPendu}, \mathrm{iVC}$ and $\mathrm{VO}_{2}$ rest are significantly lower in TP compared to $\mathrm{AB}$, mainly due to denervation of the respiratory muscles and an early onset of fatigue of the remaining innervated muscles.

\section{Acknowledgements}

The authors express their gratitude to $\mathrm{Mr} \mathrm{BF}$ van Dooren and $\mathrm{Mr}$ ACA Vissers for valuable assistance and technical help, to Dr YF Heijdra for her advice and to Dr JF Mulder for statistical help.

\section{References}

1 Arora NS, Rochester DF. Respiratory muscle failure. Med Clin North Am 1983; 67: 573-598.

2 Black LF, Hyatt RE. Maximal respiratory pressures: normal values and relationships to age and sex. Am Rev Respir Dis 1969; 99: $696-702$

3 Campbell EJM, Agostoni E, Newsom-Davis J. The respiratory muscles: mechanics and neural control. WB Saunders Co: Philadelphia, 1970; pp. 161-171.
4 Davidoff G, Schultz JS, Lieb T. Rehospitalization after initial rehabilitation for acute spinal cord injury: incidence and risk factors. Arch Phys Med Rehabil 1990; 71: 121 - 124.

5 Davis GM et al. Cardiovascular responses to arm-cranking and FNS-induced leg exercise in paraplegics. J Appl Phys 1990; 69: $671-677$.

6 De Troyer A, Heilporn A. Respiratory Mechanics in Tetraplegia: the Respiratory Function of the Intercostal Muscles. Am Rev Respir Dis 1980; 122: $591-600$.

7 Eastwood PR, Hillman DR, Finucane KE. Ventilatory responses to inspiratory threshold loading and role of muscle fatigue in task failure. J Appl Physiol 1994; 76: 185-195.

8 Estenne $\mathrm{M}$ et al. Lung volume restriction in patients with chronic respiratory muscle weakness: the role of microatelectasis. Thorax 1993; 48: 698 - 701

9 Glaser RM. Physiological aspects of spinal cord injury and functional neuromuscular stimulation. Centr Nervous Syst Trauma 1986; 3: 49-62.

10 Gross D et al. The Effect of Training on Strength and Endurance of the Diaphragm in Tetraplegia. Am J Med 1980; 68: 27-35.

11 Heydra YF et al. Differences between sniff mouth pressures and static maximal inspiratory mouth pressures. Eur Respir J 1993; 6: $541-546$.

12 Hopman MTE, Oeseburg B, Binkhorst RA. Cardiovascular responses in paraplegic subjects during arm exercise. Eur J Appl Physiol 1992; 65: $73-78$.

13 Janssen TWJ et al. Isometric strength, sprint power, and aerobic power in individuals with a spinal cord injury. Med Sci Sports Exerc 1993; 25: $863-870$.

14 Karvonen J, Saarelainen S, Nieminen MM. Measurement of Respiratory Muscle Forces Based on Maximal Inspiratory and Expiratory Pressures. Respiration 1994; 61: 28-31.

15 Koulouris $\mathrm{N}$ et al. Comparison of two different mouthpieces for the measurement of Pimax and Pemax in normal and weak subjects. Eur Respir J 1988; 1: 863-867.

16 Leech JA, Ghezzo H, Stevens D, Bechlake MR. Respiratory pressures and function in young adults. Am Rev Respir Dis 1983; 128: $17-23$.

17 Martyn JB, Moreno RH, Pare PD, Pardy RL. Measurement of Inspiratory Muscle Performance with Incremental Threshold Loading. Am Rev Respir Dis 1987; 135: 919-923.

18 Mayos M, Giner J, Casan P, Sanchis J. Measurement of Maximal Static Respiratory Pressures at the Mouth with Different Air Leaks. Chest 1991; 100: $364-366$.

19 O'Donnell DE, Sanii R, Dubo H, Loveridge B, Younes M. Steady-State Ventilatory Responses to Expiratory Resistive Loading in Quadriplegics. Am Rev Respir Dis 1993; 147: 54-59.

20 Ringqvist T. The ventilatory capacity in healthy subjects: an analysis of casual factors with special reference to the respiratory forces. Scand J Clin Invest 1966; 18: 8-170.

21 Vincken W, Ghezzo H, Cosio MG. Maximal static respiratory pressures in adults: normal values and their relationship to determinants of respiratory function. Bull Eur Physiopathol Respir 1987; 23: 435 - 439.

22 Wilson SH, Cooke NT, Edwards RHT, Spiro SG. Predicted normal values for maximal respiratory pressures in caucasian children. Thorax 1984; 39: 535-538. 